\title{
Deformation of DNA molecules by hydrodynamic focusing
}

\author{
By PAK KIN WONG ${ }^{1}$, YI-KUEN LEE AND CHIH-MING HO \\ ${ }^{1}$ Mechanical and Aerospace Engineering Department, University of California Los Angeles, \\ Los Angeles, CA 90095, USA \\ ${ }^{2}$ Department of Mechanical Engineering, The Hong Kong University of Science and Technology, \\ Clear Water Bay, Hong Kong
}

(Received 23 July 2002 and in revised form 1 July 2003)

The motion of a DNA molecule in a solvent flow reflects the deformation of a nano/microscale flexible mass-spring structure by the forces exerted by the fluid molecules. The dynamics of individual molecules can reveal both fundamental properties of the DNA and basic understanding of the complex rheological properties of long-chain molecules. In this study, we report the dynamics of isolated DNA molecules under homogeneous extensional flow. Hydrodynamic focusing generates homogeneous extensional flow with uniform velocity in the transverse direction. The deformation of individual DNA molecules in the flow was visualized with video fluorescence microscopy. A coil-stretch transition was observed when the Deborah number $(D e)$ is larger than 0.8 . With a sudden stopping of the flow, the DNA molecule relaxes and recoils. The longest relaxation time of T2 DNA was determined to be $0.63 \mathrm{~s}$ when scaling viscosity to $0.9 \mathrm{cP}$.

\section{Introduction}

A DNA molecule is a long-chain macromolecule with two polynucleotides in the form of a double helix. Each polynucleotide is composed of four types of nucleotide: adenine $(\mathrm{A})$, guanine $(\mathrm{G})$, thymine $(\mathrm{T})$ and cytosine $(\mathrm{C})$. The diameter of the double helix structure is $2 \mathrm{~nm}$ and the length of the DNA depends on the number of nucleotide base pairs. The DNA found in most human cells it contains $6 \times 10^{9}$ base pairs and has a total contour length of $2 \mathrm{~m}$. DNA can be viewed as a long and thin nanoscale mass-spring flexible structure, that experiences stretching and compression from the velocity shear found in microreactors. In solvents, DNA stays in a coiled configuration and how the small solvent molecules exchange momentum with the macro DNA molecule is a very intriguing question.

In dilute polymer solution flow (Larson 1999), the solvent velocity gradient can deform the polymer molecules from equilibrium. The interaction between polymer and solvent molecules results in the non-Newtonian properties of the flow. The viscosity of the solution can be very different from a pure solvent and it also crucially depends on the stretched state of the polymer. In more concentrated solutions, e.g. melts and gels, the interactions among the polymer molecules become important and lead to more complex material properties.

In classical theory, a flexible polymer is modelled as a series of beads connected with Gaussian springs. The chain is suspended in a viscous liquid medium subjected to a hydrodynamic drag force proportional to the velocity of the bead through the 
fluid (Doi \& Edwards 1986). Solving the linearized equations of motion yields a set of discrete relaxation times, which are proportional to the ratio between the drag coefficient and entropic elasticity of the molecule and are in the form

$$
\tau_{k}=\frac{\alpha_{k} M \mu[\mu]}{R T}
$$

where $\alpha_{k}$ is a constant dependent on index $k, M$ is the molecular weight of the molecules, $\mu$ is the solvent viscosity, $[\mu]$ is the intrinsic viscosity of the molecules, $R$ is the universal gas constant, and $T$ is the absolute temperature. Therefore, each relaxation time differs from the others by a set of constants, which are obtained by solving the characteristic value problem arising from the set of equations of motion (Zimm, Roe \& Epstein 1956). Relaxation times are the key parameters of polymer solution dynamics, as they directly correlate with different modes of molecule motion and the hydrodynamic properties of the solution. Near equilibrium, the motion of a macromolecule is best described by the longest relaxation time.

In homogeneous extensional flow, theory predicts that a coil-stretch transition occurs when the Deborah number $(D e)$, the product of the longest relaxation time $\tau_{1}$ and the strain rate of the flow $\varepsilon$, is larger than a threshold value $B$ (de Gennes 1974; Larson \& Magda 1989).

$$
D e=\varepsilon \tau_{1}>B .
$$

Physically, the relaxation time provides a time scale for the time required for a stretched polymer to recoil back to natural equilibrium. The strain rate is a time scale for the speed of the fluid that is deforming the molecules. The ratio between these two time scales provides the critical value in determining the stretching of the molecule. The stretching of the molecule is expected only when $D e$ is larger than this threshold value. In most studies, the critical values of $B$ cited for coil-stretch transitions are between 0.5 and 1 (Nguyen, Yu \& Kausch 1995; Perkins, Smith \& Chu 1997; Larson 1999).

Instead of investigating the polymer properties in a bulk continuum consisting of numerous macromolecules, direct study of a single macromolecule can provide insight into more fundamental issues of the complex system. To measure the relaxation time of a single DNA molecule, one has to first stretch the long-chain molecule from its equilibrium coiled state and then release the straining force field to observe the relaxation process. One approach is to anchor the DNA to a surface-modified micro bead and then hold the bead by magnetic tweezers (Smith, Finzi, \& Bustamante 1992) or by a laser trapping system (Perkins et al. 1994, 1995) in a flow field. The flow passes over the bead and stretches the DNA molecule. These techniques are powerful in manipulating the DNA molecules; however it is obviously of interest to study the hydrodynamic stretching without attaching beads to the molecules. Different types of flow, such as shear flow (Manneville et al. 1996; Smith, Babcock \& Chu 1999), have been applied to deform DNA molecules. However, a spatial streamwise velocity gradient (extensional flow) exerting a differential drag force along the DNA is ideal for stretching the molecule. The effect is more dramatic than with shear flow (de Gennes 1999). Experimentally, the geometries that have been used for extensional flow studies of macromolecules include: entrance flow (James \& Saringer 1982), converging flow (James 1991) and stagnation flow (Muller, Odell \& Keller 1988; Perkins et al. 1997). Among these, stagnation flow (by a cross-slot or opposed jets) has been the most commonly applied method for generating extensional flow since it was first reported (Scrivener et al. 1979). It provides homogeneous extensional flow with a significant 


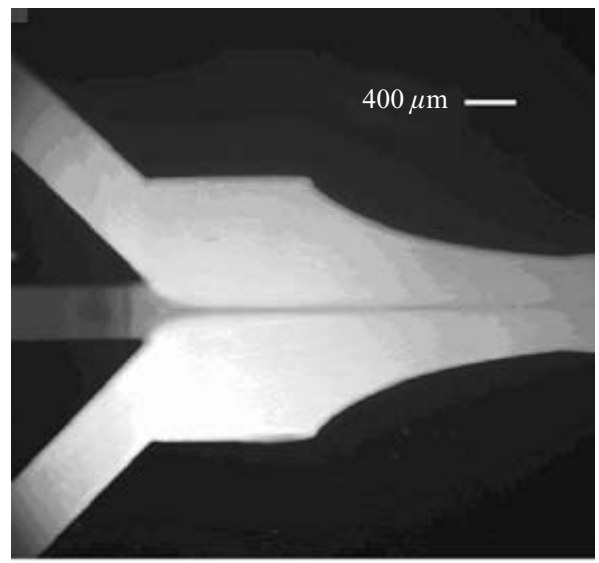

FIGURE 1. A microchannel for hydrodynamic focusing.

strain rate near the stagnation point. However, all three flows are non-uniform in the spanwise direction because of the existence of a transverse shear component due to the confining walls.

Micro-electro-mechanical-system (MEMS) technologies (see review articles by Ho \& Tai 1996, 1998, and Ho 2001) facilitate the direct study of a single macromolecule. Here, we study the dynamics of single DNA molecules under extensional flow generated by hydrodynamic focusing. Figure 1 shows the channel designed for hydrodynamic focusing. By applying two streams of a buffer solution which sandwiches the middle stream of DNA solution, hydrodynamic focusing is able to make the middle stream converge and achieve a constant spatial acceleration in the streamwise direction. This extensional flow also has a uniform velocity profile in the spanwise direction. The outer streams are fast moving and relaxe the nonslip condition dictated by solid boundaries, such that spanwise homogeneity is achieved. Therefore, the measurements are not sensitive to the initial positions of the molecules in the spanwise direction, because there is no velocity gradient in the spanwise direction. Another key advantage of using hydrodynamic focusing is that the extensional rate primarily depends only on the flow rate of the two outer fluid streams, not the middle stream containing DNA molecules. We can use a low flow rate in the middle stream so that pre-shear of DNA at the centreline is minimized while independently controlling the extensional rate using the outer channels flows. The extremely low mixing among the streams in micro flows makes independent control possible.

In this work, we study the conformational dynamics, such as the deformation and orientation, of individual T2 DNA molecules in dilute solution by hydrodynamic focusing. We first characterize the DNA solution by measuring the longest relaxation time of T2 DNA. The critical strain rate for coil-stretch transition is then determined experimentally. Finally, we report the dynamics of DNA molecules under homogeneous extensional flow and near the stream interface, where a large velocity gradient was observed due to the large velocity difference between the streams.

\section{Experimental techniques}

The experiment was performed in a $\mathrm{pH} 8$ buffer solution, containing $10 \mathrm{mM}$ TrisEDTA and $10 \mathrm{mM} \mathrm{NaCl}$. In order to reduce photobleaching and light-induced damage 


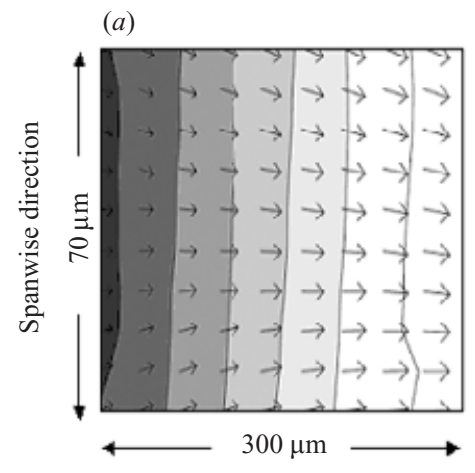

Streamwise direction

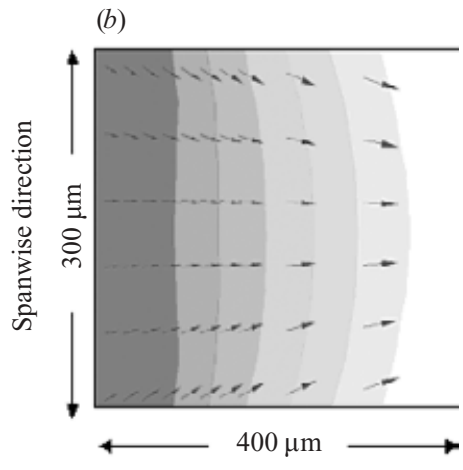

Streamwise direction

FIGURE 2. Flow field characterization in the extensional region of the hydrodynamic focusing chip. (a) PIV measurement. (b) CFD simulation. The contours in the figures represent the magnitude of the velocity fields.

to the DNA, $50 \mathrm{mg} \mathrm{ml}^{-1}$ glucose oxidase (Sigma), $<2 \mathrm{mg} \mathrm{ml}^{-1}$ catalase (Sigma), $4 \%$ beta-mercaptoethanol (Fisher) and $\sim 10 \%(\mathrm{w} / \mathrm{w})$ glucose were used to remove oxygen and free-radicals in the buffer solution (Gurrieri et al. 1997). The buffer viscosity was adjusted by addition of sucrose and measured with an Ostwald Dropping Pipet at $23^{\circ} \mathrm{C}$. T2 DNA $(164 \mathrm{kbp})$ was purchased from Sigma and stained with YOYO-1 (Molecular Probes Y-3601) at a dye to base-pair ratio of 1:5, which is expected to change the contour length from $\sim 56 \mu \mathrm{m}$ to $\sim 71 \mu \mathrm{m}$ by scaling to the number of base pairs of stained $\lambda$ DNA (Smith, Perkins \& Chu 1996). The DNA-dye complex was allowed to equilibrate for more than 1 hour before the experiment was performed.

The channel for generating hydrodynamic focusing was fabricated on a $4 \mathrm{in}$. double-sided polished silicon wafer by micromachining. The process started from photolithography of SU-8 photoresist on the wafer to define the channel geometry. The wafer was then etched by deep reactive ion etching (PlasmaTherm SLR-770) to the desired channel depth. Then photolithography was performed on the backside to define the inlets and outlets of the channel. A dummy silicon wafer was glued to the wafer before the second deep reactive ion etching. The channel was sealed by doublesided anodic bonding of Pyrex glasses. Buffer and DNA solutions were delivered into the microchannel with two digital syringe pumps (Harvard apparatus 44I/W). The buffer solution was pumped to the side channels with a T-shaped flow divider while DNA solution was pumped to the middle channel. The microchannel was loaded onto an epi-fluorescence microscope (Olympus IX70) equipped with a $100 \mathrm{~W}$ mercury lamp and a $60 \mathrm{X}$, NA 0.9 water immersion objective. The time-dependent conformation of DNA molecules was recorded on videotape by an intensified CCD camera (Hamamatsu C5909) and digitalized with a video capture card (Truevision TARGA 1000). The digital images were then analysed to extract DNA conformational information.

\section{Microchannel characterization}

A micron-resolution particle image velocimetry (PIV) system (Santiago et al. 1998) was developed to measure the velocity profile and extensional rate of the solvent flow inside the silicon channel (figure $2 a$ ). In order to make measurements with a spatial resolution of several microns and to prevent disruption of the flow field, 


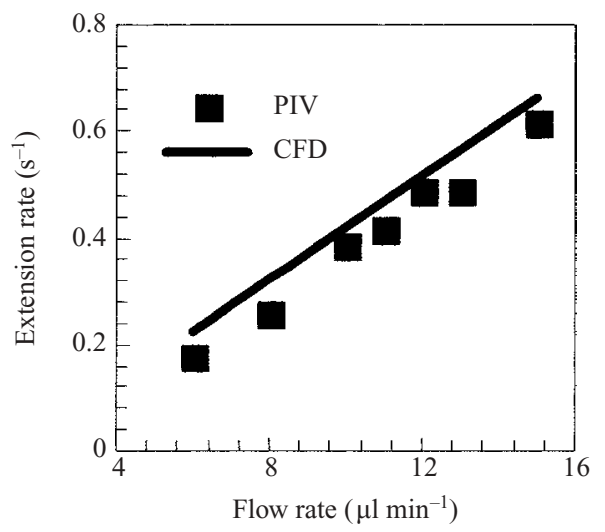

FIGURE 3. Comparison of strain rates estimated by PIV measurement and CFD simulation at different flow rates of the side streams.

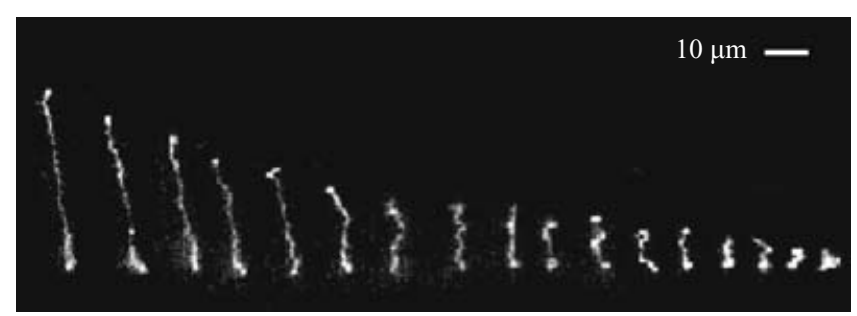

FIgURE 4. Relaxation of a DNA molecule after hydrodynamic stretching. Each image is separated by $2.5 \mathrm{~s}$ (from left to right).

the PIV experiments were carried out by seeding de-ionized water with fluorescent microspheres of diameter 300 or $500 \mathrm{~nm}$ (Duke R300, R500). We illuminated the tracers with two 5ns Nd:YAG lasers (New Wave MiniLase III-15) and captured the images with a $1024 \times 1024$ pixel, 16-bit cooled CCD camera (Photometric CH350L). Double-exposure pictures were analysed with an autocorrelation-based PIV system (TSI Inc. Insight). By averaging the PIV results for more than 20 images, we minimized the velocity uncertainty related to random Brownian motion, as Brownian noise is unbiased in nature.

We further verified the measurement by numerical simulation (CFDRC CFD$\mathrm{ACE}+$ ) (figure $2 b$ ). Figure 3 shows a comparison of the centreline strain rate from PIV measurement and a CFD calculation. The calculation and measurements were performed in a $495 \mu \mathrm{m}$ depth microchannel. The flow rate in the middle channel was kept constant at $0.5 \mu 1 \mathrm{~min}^{-1}$. The same trend was observed for channel depths from 200 to $500 \mu \mathrm{m}$.

\section{Relaxation time}

We experimentally measured the longest relaxation time of T2 DNA by stretching the molecules and observing the relaxation process after the flow was stopped (Manneville et al. 1996) (figures 4 and 5). We increased the buffer viscosity to $21.5 \mathrm{cP}$ with $50 \%(\mathrm{w} / \mathrm{w})$ glucose to improve time resolution and to enhance stretching. Relaxation times are linearly proportional to buffer viscosity (Chapman et al. 1969) and the average extension increases with buffer viscosity (Smith et al. 1999). The 


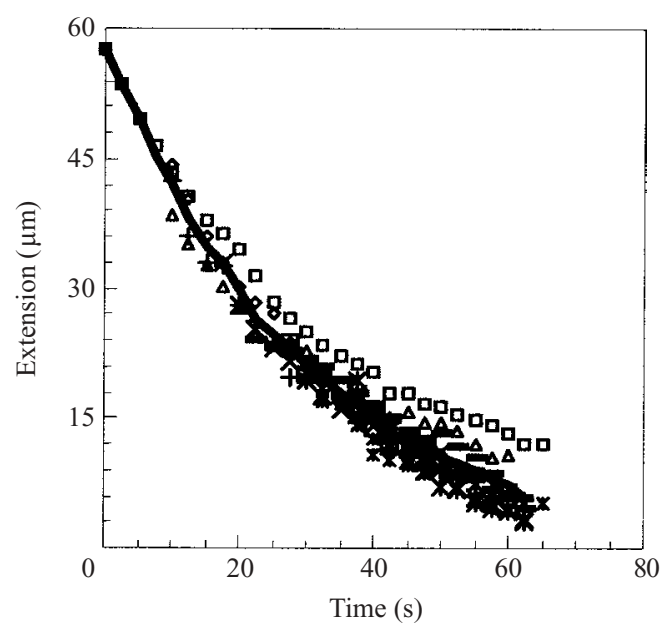

FIGURE 5. Lengths of DNA molecule during relaxation. Each symbol represents a trace of an individual molecule.

longest relaxation time was estimated by fitting the function (Perkins et al. 1997)

$$
\langle x(t) x(t)\rangle=C_{1} \mathrm{e}^{-t / \tau}+C_{2}
$$

where $C_{1}, C_{2}$ are constants, $x(t)$ is the length of the molecule at time $t, \tau$ is the relaxation time of fluid stress. This relation is expected to be valid near equilibrium, where the stress in the fluid is linearly proportional to $\langle x(t) x(t)\rangle$ and the contribution of higher-order relaxation modes should be negligible. The stress relaxation time of the fluid near equilibrium could be approximated as the longest relaxation time of the molecules.

The longest relaxation time of T2 DNA at these conditions is determined to be $15.0 \mathrm{~s}$ by averaging over 16 individual molecules. To compare our result with values obtained by other methods, we scaled viscosity to $0.9 \mathrm{cP}$, which gave the longest relaxation time to be $0.63 \mathrm{~s}$. The result is somewhat higher than the relaxation time of $0.52 \mathrm{~s}$ predicted by theory (Zimm 1956; Zimm et al. 1956) and $0.45 \mathrm{~s}$ in birefringence measurements (Thompson \& Gill 1967). We calculated the relaxation time for a non-free-draining coil using the equation from Zimm with an intrinsic viscosity of $290 \mathrm{dl} \mathrm{g}^{-1}$ and a molecular weight of $1.1 \times 10^{8}$ Daltons (Chapman et al. 1969). The difference is mainly due to the increase in contour length and persistence length caused by the fluorescent labelling of DNA and a possible difference in ionic concentration in the buffer solution. On the other hand, it is in reasonable agreement with the $0.65 \mathrm{~s}$ obtained for YoYo-1 labelled $\lambda$ DNA by using a scaling exponent of 1.66 (Perkins et al. 1994).

\section{DNA molecules in homogeneous extensional flow}

We succeeded in obtaining a spanwise uniform extensional flow when two side streams merge with the middle stream in the microchannel. The flow is accelerating in the streamwise direction while the velocity is constant along the spanwise direction for at least $70 \mu \mathrm{m}$ in our imaging area. This unique flow is achieved by taking advantage of the low mixing rate at the interface between the streams. In flows at low Reynolds number (0.01-0.1), molecular diffusion is the only natural mechanism for mixing across streams and it is so slow that a fairly long streamwise distance is achievable 


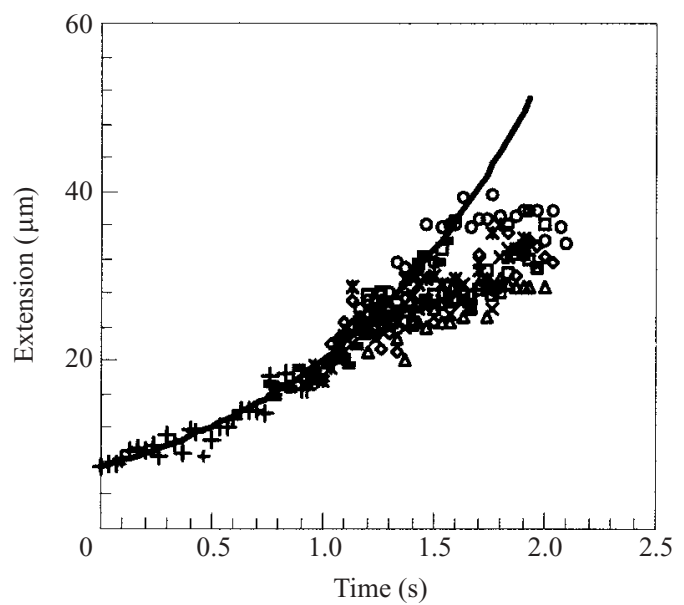

FIGURE 6. Lengths of DNA molecules as a function of time inside the extensional region. Each symbol represents a trace of an individual molecule. The curves are shifted in time to compare with the fluid deformation estimated by the PIV measured velocity field (solid line).

for the study. The Reynolds number is defined as

$$
R e=\frac{\rho V L}{\mu}
$$

where $\rho$ is density of the solution, $V$ represents the velocity of the DNA solution and $L$ the length of uniform velocity in the spanwise direction of the flow.

We fixed the flow rate in the middle stream to $0.5 \mu 1 \mathrm{~min}^{-1}$ and controlled the streamwise strain rate by adjusting the flow rate in the side channels. The extensional rate is independent of the middle stream flow rate over the range we have measured and has a linear relationship with the flow rate of the two side channels (figure 3). Minimizing the initial velocity in the middle stream allows a larger dynamic range in our study and minimizes pre-shear before the DNA enters the accelerating regions. All the measurements were performed in the middle of the channel where the molecules are subject to zero shear stress. We measured the DNA deformation in the region near the centreline to ensure uniform streamwise stretching rate. The viscosity of the buffer solution was $1.14 \mathrm{cP}$.

To obtain the threshold value, $B$, for coil-stretch transition, $D e$ was gradually adjusted by increasing the flow rate of the side channels, which controlled the velocity gradient experienced by the molecules. At $D e \sim 0.8$, stretching of some DNA molecules was suddenly observed. It should be noted that we observed a scatter in residency time, the time the DNA molecules experience the acceleration field before they were stretched. Some of the molecules started to deform upon entering the extensional region while some were not stretched within the entire imaging area. This is consistent with the observation from Perkins et al. (1997) - coil-stretch transition is not a simultaneous process for all the molecules when the Deborah number reaches the threshold value, which is 0.8 in the present case. This is probably due to the range of conformational states of DNA molecules when encountering the velocity gradient. The instantaneous conformation of DNA dictated the hydrodynamic force experienced by the molecule; and therefore the onset of stretching.

From the micro-PIV measured velocity field, the fluid deformation can be obtained as a function of time. We then compared the fluid deformation with the DNA strained by the flow (figure 6). The DNA molecule follows the fluid deformation at small strain 


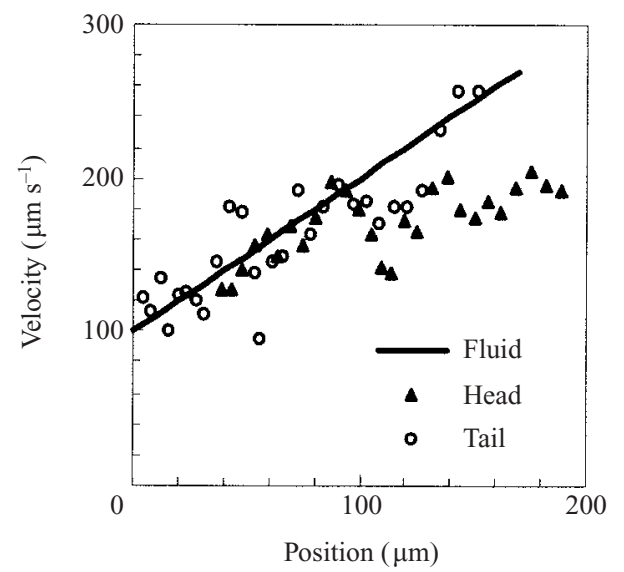

FiguRE 7. Tracking of head and tail velocities of a DNA molecule. The solid line indicates the fluid velocity at different position in the streamwise direction.

rates but deviates at large extension. At $D e \sim 0.8$, deviations from fluid deformation occur roughly at $25 \mu \mathrm{m}$ extension for T2 DNA, which is approximately $35 \%$ of its contour length. Further physical insight can be obtained by tracking both ends (head and tail) of the DNA molecules. We estimated the velocities of the ends of the DNA molecules and compared them with the fluid velocity, which increases linearly with spatial position (figure 7). At small deformation, both ends have the same velocity as the fluid at the corresponding position. This is because the hydrodynamic drag force dominates the elastic force of the molecules. Each DNA segment responds independently and changes in the same way as the macroscopic solvent, which is often referred as affine deformation (Smith \& Chu 1998). This result also indicates that the non-slip boundary condition is appropriate for the DNA molecules in this experimental condition. This is interesting since the non-slip boundary condition was developed for continuum fluids but it is still applicable for the present case. The reason is that the observed phenomenon is due to the averaged momentum exchanges over a large number of small solvent molecules. Affine deformation is not expected for highly extended molecules because the drag force is no longer the dominant force in such conditions (Doi \& Edwards 1986). As shown in figure 7, the velocity of the fast end (head) dropped below that of the fluid after about $100 \mu \mathrm{m}$, whereas the velocity of the tail roughly follows that of the fluid. The fast head was slowed down by the slow tail due to the elastic force and the molecule starts to deviate from the fluid deformation.

\section{DNA deformation in the velocity shear region}

To study the response of DNA molecules near the stream interface, we measured the velocity distribution near the stream interface by tracking undeformed molecules and compared the results with CFD calculations (figure 8). Because of the high velocity difference between fluid streams, a discontinuity in velocity was observed at the location where two streams merge. The discontinuity quickly develops into a high velocity gradient along streamlines close to the interface. The velocity gradient displays a strong dependence on the initial position. In general, an initial position closer to the channel sidewall has a larger velocity gradient. For an initial position more than $\sim 50 \mu \mathrm{m}$ from the channel sidewall, the velocity gradient approaches the 

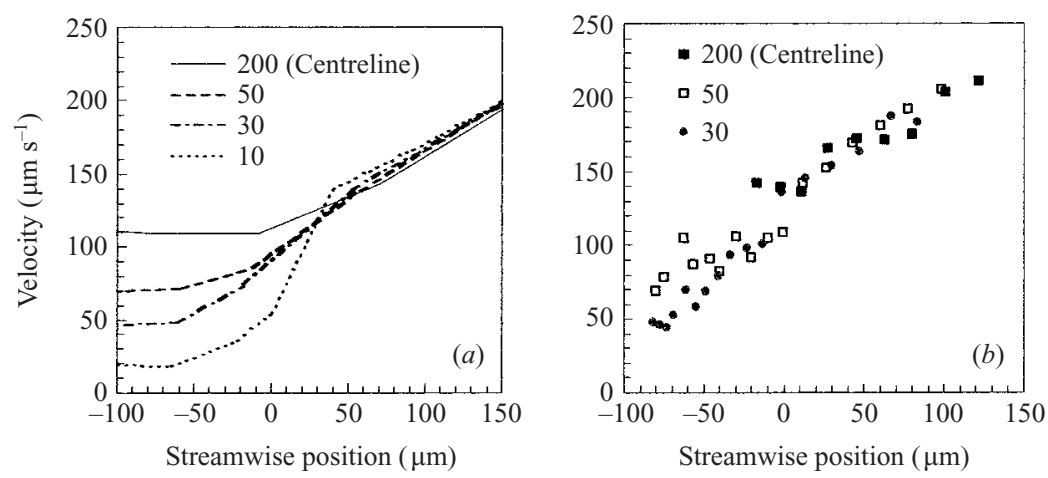

FIGURE 8. Velocities in the streamwise direction estimated by $(a)$ CFD and $(b)$ tracking DNA molecules. The curves represents various initial positions from the channel side wall (in $\mu \mathrm{m}$ ).

value near the centreline. After merging to the extensional region, accelerations are roughly the same along all the streamlines and are independent of initial position. Both DNA tracking and CFD calculations show similar behaviour near the stream interface.

We observed a higher probability of molecules being stretched near the interface. This is partially because the fluid stream near the interface has a higher velocity gradient. On the other hand, DNA near the channel sidewall experienced pre-shear before entering the interface. In our experiment, we set the flow rate of the middle channel to be $0.5 \mu 1 \mathrm{~min}^{-1}$ which gave the value of the Weissenberg number (We), the product of longest relaxation time and the static shear rate, to be $\sim 2$ at the sidewall. At this $W e$, the average deformations are small compared to the contour length; however, the fluctuations in length could deviate far from the natural equilibrium (Smith et al. 1999). It has been reported that shear flow tends to align DNA molecules towards the flow direction (LeDuc et al. 1999) and this alignment can enhance the stretching as it can maximize the velocity difference experienced by the molecule under the velocity gradient.

\section{Concluding remarks}

Hydrodynamic focusing can be used to study the dynamics of an individual DNA molecule stretched far from its natural equilibrium. Our results have measured the relaxation time of T2 DNA molecules. Micro-flow channels with streams of different velocities can provide streamwise extensional flow with uniformity in the transverse direction. This unique capability yielded interesting results in the deformation of a long-chain coiled molecule under stretching by solvent molecules and the applicability of the non-slip condition derived from the fluid continuum concept.

While efforts can be focused on understanding the fundamentals of macromolecule deformation, single molecule manipulation techniques using hydrodynamic force are also invaluable in molecular biotechnology. For instance, our microchannel is potentially a bioreactor for enhancing bio-reaction of DNA molecules. Stretching of DNA molecules can increase the effective contact area for easier hybridization with oligo probes. This feature can significantly aid in bio-signature detection.

The authors would like to acknowledge Professor Chiang Shih at Florida State University for his insightful suggestion. This work is supported by DARPA Bioflips project. 


\section{REFERENCES}

Chapman, R. E., Klotz, JR., L. C., Thompson, D. S. \& Zimm, B. H. 1969 An instrument for measuring retardation times of deoxyribonucleic acid solutions. Macromolecules 2 , 637-643.

DoI, M. \& Edwards, S. F. 1986 The Theory of Polymer Dynamics. Clarendon.

DE GenNes, P. G. 1974 Coil-stretch transition of dilute flexible polymers under ultrahigh velocity gradients. J. Chem. Phys. 60, 5030-5042.

DE Gennes, P. G. 1999 Molecular individualism. Science 276, 1999-2000.

Gurrieri, S., Wells, K. S., Johnson, I. \& Bustamante, D. C. 1997 Direct visualization of individual DNA molecules by fluorescence microscopy: characterization of the factors affecting signal/background and optimization of imaging conditions using YoYo. Analyt. Biochem. 249, 44-53.

Happel, J. \& Brenner, H. 1965 Low Reynolds Number Hydrodynamics with Special Applications to Particulate Media. Prentice-Hall.

Ho, C. M. 2001 Fluidics - the link between micro and nano sciences and technologies. Tech. Digest of the 14th IEEE Intl MEMS Conf., Interlaken, Switzerland, pp. 375-384 (ISBN-0-78036251-9).

Ho, C. M. \& TAI, Y. C. 1996 MEMS and its applications for flow control. Trans. ASME: J. Fluids Engng 118, 437-447.

Ho, C. M. \& TAI, Y. C. 1998 Micro-electro-mechanical-systems and fluid flows. Annu. Rev. Fluid Mech. 30, 579-612.

JAMEs, D. F. 1991 Flow in a converging channel at moderate Reynolds numbers. AIChE J. 37, 59-64.

JAmes, D. F. \& SARINGer, J. H. 1983 Flow of dilute polymer solution through converging channels. J. Non-Newtonian Fluid Mech. 11, 317-339.

LARSON, R. G. 1999 The Structure and Rheology of Complex Fluids. Oxford.

LARson, R. G. \& MaGdA, J. J. 1989 Coil-stretch transition in mixed shear and extensional flows of dilute polymer solutions. Macromolecules 22, 3004-3010.

LeDuc, P., Haber, C., Bao, G. \& Wirtz, D. 1999 Dynamics of individual flexible polymer in a shear flow. Letters to Nature 399, 564-566.

Macosko, C. W. 1994 Rheology: Principles, Measurements and Applications. VCH, New York.

Manneville, S., Cluzel, PH., Viovy, J. L., Chatenay, D. \& Caron, F. 1996 Evidence for the universal scaling behaviour of a freely relaxing DNA molecule. Europhys. Lett. 36, 413-418.

Muller, A. J., Odell, J. A. \& Keller, A. 1988 Elongation flow and rheology of monodisperse polymer in solution. J. Non-Newtonian Fluid Mech. 30, 99-118.

Nguyen, T. Q., Yu, G. \& Kausch, H. 1995 Birefringence of a polystyrene solution in elongational flow: effects of molecular weight and solvent quality. Macromolecules 28, 4851-4860.

Perkins, T. T., Quake, S. R., Smith, D. E. \& Chu, S. 1994 Relaxation of a single DNA molecule observed by optical microscopy. Science 264, 822-826.

Perkins, T. T., Smith, D. E. \& ChU, S. 1997 Single polymer dynamics in an elongational flow. Science 276, 2016-2021.

Perkins, T. T., Smith, D. E., Larson, R. G. \& Chu, S. 1995 Stretching of a single tethered polymer in a uniform flow. Science 268, 83-87.

Santiago, J. G., Wereley, S. T., Meinhart, C. D., Beebe, D. J. \& Adrian, R. J. 1998 A particle image velocimetry system for microfluidics. Exps. Fluids 25, 316-319.

Scrivener, O., Bernner, C., Cressely, R., Hocquart, R., Sellin, R., \& Vlachos, N. S. 1979 Dynamical behaviour of drag-reducing polymer solutions. J. Non-Newtonian Fluid Mech. 5, 475-495.

Sмith, D. E., BaвCock, H. P. \& Chu, S. 1999 Single-polymer dynamics in steady shear flow. Science 283, 1724-1727.

Smith, D. E. \& Chu, S. 1998 Response of flexible polymers to a sudden elongational flow. Science 281, 1335-1340.

Smith, D. E., Perkins, T. T. \& ChU, S. 1996 Dynamical scaling of DNA diffusion coefficients. Macromolecules 29, 1372-1373.

Smith, S. B., Finzi, L. \& Bustamante, C. 1992 Direct mechanical measurements of the elasticity of single DNA molecules by using magnetic beads. Science 258, 1122-1126. 
Thompson, D. S. \& Gill, S. J. 1967 Polymer relaxation times from birefringence relaxation measurements. J. Chem. Phys. 47, 5008-5017.

Zıмm, B. H. 1956 Dynamics of polymer molecules in dilute solution: viscoelasticity, flow birefringence and dielectric loss. J. Chem. Phys. 24, 269-278.

Zimm, B. H., Roe, G. M. \& Epstein, L. F. 1956 Solution of a characteristic value problem from the theory of chain molecules. J. Chem. Phys. 24, 279-280. 